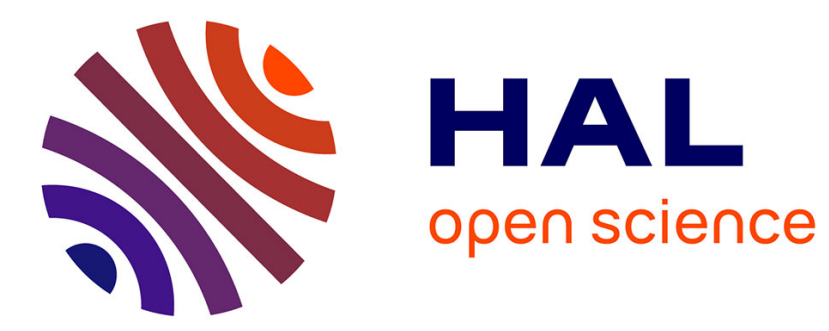

\title{
Capillary descent
}

Joachim Delannoy, Hélène de Maleprade, Christophe Clanet, David Quéré

\section{To cite this version:}

Joachim Delannoy, Hélène de Maleprade, Christophe Clanet, David Quéré. Capillary descent. Soft Matter, 2018, 14 (26), pp.5364-5368. 10.1039/c8sm00453f . hal-02356906

\section{HAL Id: hal-02356906 https://hal.science/hal-02356906}

Submitted on 7 Jan 2021

HAL is a multi-disciplinary open access archive for the deposit and dissemination of scientific research documents, whether they are published or not. The documents may come from teaching and research institutions in France or abroad, or from public or private research centers.
L'archive ouverte pluridisciplinaire HAL, est destinée au dépôt et à la diffusion de documents scientifiques de niveau recherche, publiés ou non, émanant des établissements d'enseignement et de recherche français ou étrangers, des laboratoires publics ou privés. 


\section{Accepted Manuscript}

This article can be cited before page numbers have been issued, to do this please use: J. Delannoy, H. de Maleprade, C. Clanet and D. Quéré, Soft Matter, 2018, DOI: 10.1039/C8SM00453F.

\section{Soft Matter}

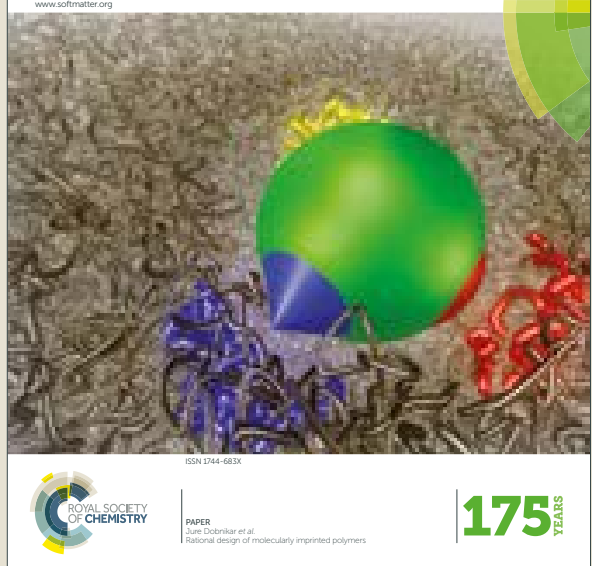

This is an Accepted Manuscript, which has been through the Royal Society of Chemistry peer review process and has been accepted for publication.

Accepted Manuscripts are published online shortly after acceptance, before technical editing, formatting and proof reading. Using this free service, authors can make their results available to the community, in citable form, before we publish the edited article. We will replace this Accepted Manuscript with the edited and formatted Advance Article as soon as it is available.

You can find more information about Accepted Manuscripts in the author guidelines.

Please note that technical editing may introduce minor changes to the text and/or graphics, which may alter content. The journal's standard Terms \& Conditions and the ethical guidelines, outlined in our author and reviewer resource centre, still apply. In no event shall the Royal Society of Chemistry be held responsible for any errors or omissions in this Accepted Manuscript or any consequences arising from the use of any information it contains. 


\title{
Capillary Descent
}

\author{
Joachim Delannoy, Hélène de Maleprade, Christophe Clanet \& David Quéré \\ Physique \& Mécanique des Milieux Hétérogènes, UMR 7636 du CNRS, ESPCI, 75005 Paris, France. \\ and \\ LadHyX, UMR 7646 du CNRS, École polytechnique, 91128 Palaiseau, France.
}

\begin{abstract}
A superhydrophobic capillary tube immersed in water and brought in contact with the bath surface will be invaded by air, owing to its aerophilicity. We discuss this phenomenon where the ingredients of classical capillary rise are inverted, which leads to noticeable dynamical features. (1) The main regime of air invasion is linear in time, due to the viscous resistance of water. (2) Menisci in tubes with millimetre-size radii strongly oscillate before reaching their equilibrium depth, a consequence of inertia. On the whole, capillary descent provides a broad variety of dynamics where capillary effects, viscous friction and liquid inertia all play a role.
\end{abstract}


Capillary rise occurs when a narrow tube is brought in contact with a wetting liquid, as first reported by Leonardo and quantified by Hauksbee and Jurin at the beginning of the $18^{\text {th }}$ century. Despite the efforts of Newton, the phenomenon was only understood one century later [1]. The meniscus is driven by a negative Laplace pressure $\Delta P=-2 \gamma \cos \theta / r$, denoting $\gamma$ as the surface tension of the liquid, $\theta<90^{\circ}$ as the contact angle and $r$ as the tube radius (assumed to be smaller than the millimeter-size capillary length $\kappa^{-1}$ ). The rise stops at the height $H$ where $\Delta P$ balances the hydrostatic pressure loss $-\rho g H$ (with $\rho$ the liquid density, and $g$ the acceleration of gravity), which classically yields:

$$
H=\frac{2 \gamma \cos \theta}{\rho g r}
$$

Considering water and assuming $\theta=0^{\circ}$ (as observed on clean glass), $H$ is expected (and found) to be typically $10 \mathrm{~cm}$ for $r=100 \mu \mathrm{m}$ - a spectacular distance responsible for the early interest on this question: confinement amplifies the natural meniscus height $\kappa^{-1}=(\gamma / \rho g)^{1 / 2}$ by a factor $\kappa^{-1} / r$. The rise dynamics was described by Bell and Cameron (1906), followed by Lucas (1918) and Washburn (1921) [2-4]. As long as the meniscus position $z$ is small compared to $H$, the meniscus speed $\dot{z}$ generally results from a balance between the capillary force $F_{\gamma}=2 \pi r \gamma \cos \theta$ and the Poiseuille friction $F_{\eta}=8 \pi \eta z \dot{z}$ [5], denoting $\eta$ as the liquid viscosity and defining $\dot{z}$ as the fluid flux per cross sectional area. This leads to the Washburn law:

$$
z^{2}(t)=\frac{\gamma r \cos \theta}{2 \eta} t
$$

The meniscus slows down as it progresses $(\dot{z} \sim 1 / z)$, a consequence of viscous friction. Later, as the final height $H$ is approached, gravity also opposes the rise and $Z$ exponentially relaxes to $H$ with a characteristic time $\eta H / \rho g r^{2}$. Interestingly, the typical time $\eta H^{2} / \gamma r \cos \theta \sim \eta H / \rho g r^{2}$ needed to reach the height $H$ following (2) obeys the same scaling as the relaxation, showing that the two phases of the rise (parabolic regime, exponential relaxation) have comparable timescales. The scaling in $t^{1 / 2}$ resists many configurations. Hele-Shaw cells, grooves or rough solids are generally invaded with such dynamics [6-7], which is also observed when complex porous media (fabrics, sand, sponges, papers) contact a wetting liquid [8-11]. This universality was exploited to determine either contact angles or characteristic radii in porous media [12-13]. 
Conversely, a few uncommon situations that escape Washburn law were recently evidenced. (i) Staple (2001), Reyssat (2008) and Figliuzzi (2013) analytically and experimentally studied the progression of liquids in capillary tubes with non-uniform cross-sections [14-16]. They reported scaling laws of the type $z(t) \sim t^{\beta}$, where $\beta$ was found to depend on the tube shape and to vary between 0 and 1/2. (ii) V-shaped channels (for which there is no more characteristic distance such as its radius for a tube) also generate special dynamics [17-19], such as $z(t) \sim t^{1 / 3}$ [20]. (iii) As recently shown by Kim (2017), porous media with largely-distributed pores also have unusual dynamics of impregnation (as $t^{1 / 4}$ ), due to the coexistence of characteristic length scales [21]. (iv) A final case concerns inertial effects at short times, especially for wide tubes: the invading liquid is then resisted by inertia and the balance of the capillary force $F_{\gamma}$ with the momentum variation $\mathrm{d}\left(\pi r^{2} \rho z \dot{z}\right) / \mathrm{d} t$ leads to a surprisingly simple law, $z(t)=c t$, where $c=(2 \gamma \cos \theta / \rho r)^{1 / 2}$ is called the inertio-capillary speed [22-23].

For non-wetting liquids $\left(\theta>90^{\circ}\right)$, Equation 1 predicts negative values for the equilibrium position of the meniscus, a situation that can be generated by bringing an "aerophilic" tube immersed in water in contact with air. Then we expect the meniscus separating air and water to be pulled down to the depth $H$ - as observed by Jurin for quicksilver in a glass tube [24]. Aerophilic tubes are obtained by coating the inner walls of tube with a hydrophobic texture, which leads to the well-known situation of superhydrophobicity, where solids are wet by air rather than by water [25-26]. In our experiments, we use glass capillary tubes with radius $r$ and total length $L$ respectively ranging between $0.51 \pm 0.01 \mathrm{~mm}$ and $1.50 \pm 0.01 \mathrm{~mm}$, and between $5.5 \pm 0.1 \mathrm{~cm}$ and $13.5 \pm 0.1 \mathrm{~cm}$. The tubes are treated by filling them with isopropanol containing silanized silica nanobeads with diameter $30 \mathrm{~nm}$ (Glaco, Soft99). After removing the solvent with compressed air, the nanocoating is consolidated at $180^{\circ} \mathrm{C}$ for $30 \mathrm{~min}$. We repeat the treatment three times to make the aerophilic layer more robust. The resulting tubes are transparent, and their walls have a typical roughness of $100 \mathrm{~nm}$, much smaller than the tube radius, as shown in the Supplementary Information (figure SI-1). The advancing and receding contact angles of water on a flat surface with the same coating are found to be $165 \pm 1^{\circ}$ and $160 \pm 2^{\circ}$, which provides for air on immersed solids the complementary values $\theta_{r}=15 \pm 1^{\circ}$ and $\theta_{a}=20 \pm 2^{\circ}$. The coated tubes are then vertically immersed at room temperature (between $20^{\circ} \mathrm{C}$ and $22^{\circ} \mathrm{C}$ ) inside a pool of deionised 
water (surface tension $\gamma=72.0 \pm 0.5 \mathrm{mN} / \mathrm{m}$, density $\rho=998 \pm 1 \mathrm{~kg} / \mathrm{m}^{3}$, viscosity $\eta=0.98 \pm$ $0.03 \mathrm{mPa} . \mathrm{s}$ ). By connecting the outer extremity of the capillary tube to a small funnel, we pump the air trapped inside with a syringe, and we visually control that no air remains. Then tubes are slowly brought up to the bath surface, using a micrometric screw (figure 1a). Contact with air defines the origin of time, from which we follow the position of the air/water interface with a high-speed video camera (Phantom v7). As seen in figures $1 \mathrm{~b}$ and $1 \mathrm{c}$, the meniscus moves downward and follows two types of dynamics: either the descent is roughly linear in time before relaxing to equilibrium (thin tubes, figure $1 \mathrm{~b}$ ), or the meniscus oscillates before reaching equilibrium (wide tubes, figure 1c). We also observe that the final position is deeper when confinement is stronger.

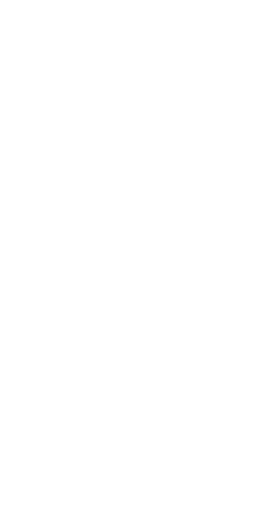

(a)

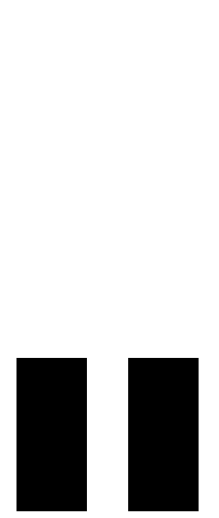

$t=280 \mathrm{~ms}$
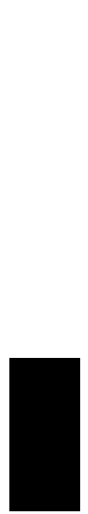

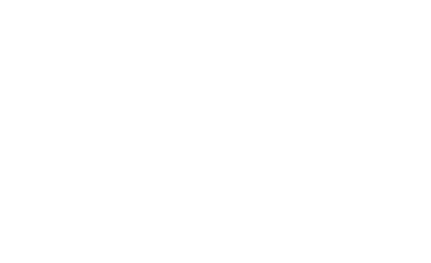

(b)

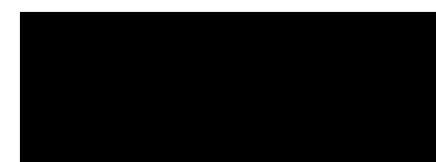

(c)

Figure 1. (a) Sketch of an experiment of capillary descent in an aerophilic tube. Blue and white represent water and air, respectively. The origin of time is taken when the tube contacts air. (b) Meniscus position at $t=50 \mathrm{~ms}, 90 \mathrm{~ms}$ and $250 \mathrm{~ms}$ for a tube with $r=0.51 \mathrm{~mm}$ and $L=7.5 \mathrm{~cm}$. Water appears in black, and air in white. The meniscus dynamics can be reconstructed from such images, and its depth $z$ is linear in time (after a short regime of acceleration) before relaxing to equilibrium. (c) Same experiment with $r=1.50$ $\mathrm{mm}$ and $L=7.4 \mathrm{~cm}$. The three pictures are taken at $t=40 \mathrm{~ms}, 280 \mathrm{~ms}$ and $1600 \mathrm{~ms}$, and the dynamics is now found to be non monotonous, with strong oscillations before equilibrium is reached.

We first discuss the centimetre-size equilibrium depth reached by the meniscus. To that end, we extract from the movies the evolution $z(t)$ of the position of the menisci for four tubes having the same length yet different radii. We plot the collection of graphs in figure 2 where dotted lines indicate the final depth expected from equation (1). 


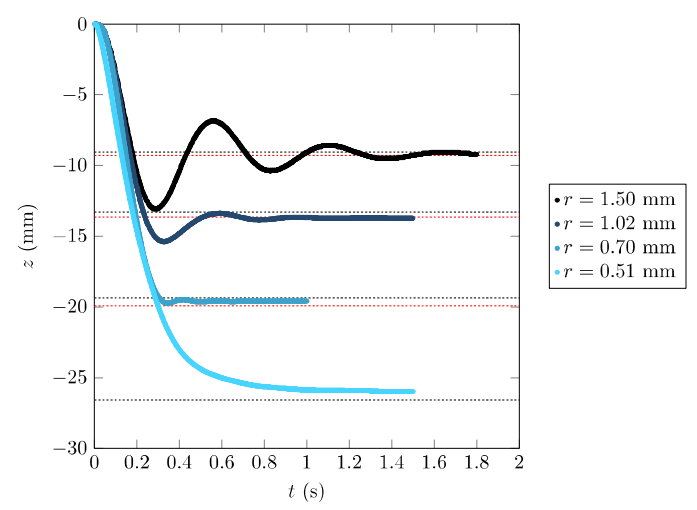

Figure 2. Meniscus dynamics in a vertical superhydrophobic tube immersed in water, after its contact to the surface of the bath. Data correspond to four tubes with the same length $(L=7.5 \pm 0.1 \mathrm{~cm})$ but different radii between $r=0.51 \mathrm{~mm}$ (light blue) and $r=1.50 \mathrm{~mm}$ (black). The dotted lines show the equilibrium depths given by equation 1 , taking either the advancing angle of air in water $\theta_{a}=20^{\circ}$ (black lines) or the receding angle $\theta_{\mathrm{r}}=15^{\circ}$ (red lines).

The agreement is good, and it can be improved by noticing that the final depth slightly depends on the dynamics. (i) At small $r(r=0.51 \mathrm{~mm})$, equilibrium is reached monotonously, so that we must compare data with the value $H\left(\theta_{\mathrm{a}}\right)$ calculated in equation 1 with the advancing angle $\theta_{\mathrm{a}}$ (black dotted line). The observed depth is smaller by $4 \%$ than expected, which might be due to coating defects. Superhydrophobic treatment at small $r$ is challenging because it must remain of homogeneous quality despite both the confinement and the large distance $z$. Coating heterogeneities make tubes less aerophilic, and thus limit the depth reached by air. Conversely, our experiment provides a simple test for checking the quality of aerophilic treatments in confined geometries. (ii) At large $r$, capillary descent is accompanied by oscillations, so that the contact angle switches between its advancing and receding values. Thus, the equilibrium depth is ill-defined between $H\left(\theta_{\mathrm{a}}\right)$ and $H\left(\theta_{\mathrm{r}}\right)$, whose theoretical values are drawn in black and red, respectively. We observe that oscillating menisci systematically reach a position between $H\left(\theta_{\mathrm{a}}\right)$ and $H\left(\theta_{\mathrm{r}}\right)$, as expected from this discussion. The uncertainty on $H$ remains modest, owing to the small angle hysteresis $\Delta \theta=\theta_{\mathrm{a}}-\theta_{\mathrm{r}}$ in our tubes (about $\left.5^{\circ}\right)$ : the relative difference between $H\left(\theta_{\mathrm{a}}\right)$ and $H\left(\theta_{\mathrm{r}}\right)$ is found in equation 1 (at small $\theta$ ) to be $\Delta \theta \cdot \bar{\theta}$ (noting $\bar{\theta}$ the mean contact angle), about $3 \%$ in our experiments.

We now discuss the dynamics of the descent, and distinguish the two kinds of behaviour (monotonic, oscillatory). As seen in figure 2 (light blue data), the meniscus in a thin tube 
is first accelerated (on a short time, smaller than $50 \mathrm{~ms}$ ) until it reaches a constant velocity of the order of $10 \mathrm{~cm} / \mathrm{s}$. Later, the meniscus relaxes to its equilibrium position $H \approx-26 \mathrm{~mm}$ in a timescale of a fraction of a second, comparable to that of the descent.

A regime of constant speed markedly differs from what is observed for a liquid rising in a tube (equation 2) [2-4]. While rising columns of liquid gradually increase in height, capillary descent consists of replacing a column of water by air of negligible viscosity. Viscous effects should mainly concern the water column in which the time necessary to establish a Poiseuille flow is $\tau_{i} \approx \rho r^{2} / 8 \eta$, that is, $\tau_{i} \approx 30 \mathrm{~ms}$ for $r=0.51 \mathrm{~mm}$. After this short inertial phase, but still far from equilibrium $(z<H)$, the force $F_{\gamma}=2 \pi r \gamma \cos \theta$ driving the meniscus is balanced by the Poiseuille friction in water $F_{\eta}=8 \pi \eta L \dot{z}$, assuming $z<<-$ a condition fulfilled for our tubes, all longer than $H$. Hence we deduce that the meniscus progresses at a constant velocity given by:

$$
V=\frac{\gamma \cos \theta}{4 \eta} \frac{r}{L}
$$

The speed of descent is determined on the one hand by the materials properties (surface tension $\gamma$, angle $\theta$, viscosity $\eta$ ), and on the other hand by the tube dimensions (radius $r$, length $L$ ). For water, $r \approx 1 \mathrm{~mm}$ and $L \approx 10 \mathrm{~cm}$, we expect $V$ to be on the order of $10 \mathrm{~cm} / \mathrm{s}$, as indeed observed in figures 1 and 2 . The characteristic time of descent $\tau_{d}=H / V$ is $8 \eta L / \rho g r^{2}$. For the thinnest tube in figure 2 , this time is $0.24 \mathrm{~s}$, in excellent agreement with the observations. Since $V$ characterizes the beginning of the descent, it is independent of $g$, which we checked by inclining the immersed tubes (figure SI-2).

We test this original visco-capillary regime by varying the aspect ratio $r / L$ of the tubes. To that end, we measure the descent velocity $V$ at the inflection point in the curves $z(t)$, that is, between the acceleration and relaxation phases. We display in figure $3 a$ these velocities as a function of the tube length $L$, at fixed $r$. As predicted by equation $3, V$ decreases in a hyperbolic way (thin lines) with $L$. Data with various $r$ and $L$ are collected in figure $3 \mathrm{~b}$, where we plot the descent velocity as a function of the aspect ratio $r / L$. All data collapse on a common straight line of slope $16.0 \pm 0.5 \mathrm{~m} / \mathrm{s}$, in good agreement with the value expected from equation $3, \gamma \cos \theta / 4 \eta \approx 17.3 \mathrm{~m} / \mathrm{s}$. 


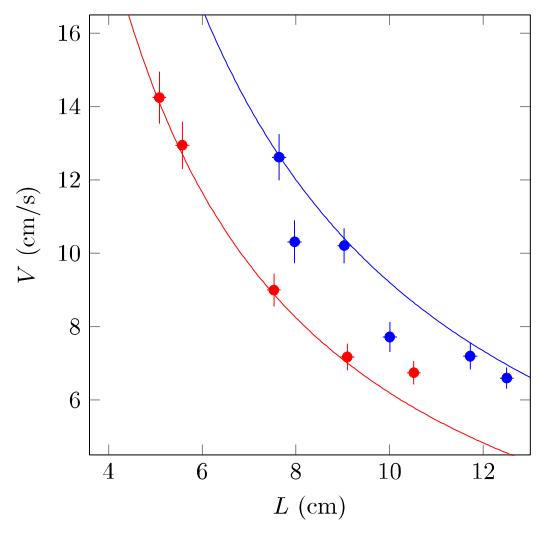

(a)

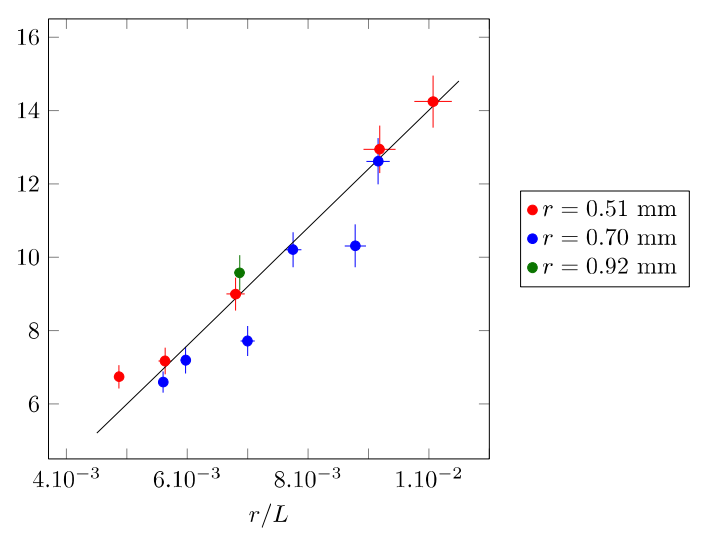

(b)

Figure

length $i$ the hyp

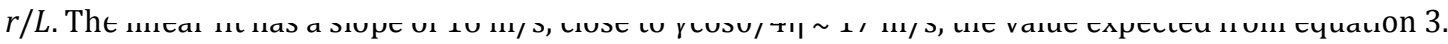

he total is show ct ratio

The relaxation following the linear descent is quite similar to that observed in capillary rise [27], and we provide some details about it in the SI (figure SI-3). Its duration is obtained after introducing the hydrostatic thrust $-\pi r^{2} \rho g z$ in the model, which yields an exponential relaxation of characteristic time $\tau=8 \eta(L-H) / \rho g r^{2}$. This time is expected to be $\sim 0.2 \mathrm{~s}$ for the thinnest tubes, in agreement with the observations in figure 2 . For long tubes $(H \ll L), \tau$ is similar to the time of descent $H / V=8 \eta L / \rho g r^{2}$ deduced from equations 1 and 3, a common feature with Washburn law, as discussed after equation 2 .

For wide tubes $(r>1 \mathrm{~mm})$, inertial effects cannot be neglected, as seen in figure 2 where the meniscus significantly overcomes the equilibrium depth and then oscillates, a behaviour also observed in capillary rise [22, 27]. Close to equilibrium $(z=H+\varepsilon, \varepsilon<<H)$, viscous friction, gravity force and inertia of water respectively scale as $\eta(L-H) \dot{\varepsilon}, \rho g r^{2} \varepsilon$ and $\rho r^{2}(L-H) \ddot{\varepsilon}$. Prefactors in these expressions can be easily calculated and the balance of forces writes: $\rho g r^{2} \varepsilon+8 \eta(L-H) \dot{\varepsilon}+\rho r^{2}(L-H) \ddot{\varepsilon}=0$. This second order differential equation results in oscillatory regimes for tube radius $r$ larger than $\left(16 \eta^{2}(L-H) / \rho^{2} g\right)^{1 / 4}-$ a quantity of $\sim 1 \mathrm{~mm}$ for water and decimeter-long tubes, as observed in figure 2 . This condition can also be expressed through a critical tube length: 


$$
L_{c}=g\left(\frac{\rho r^{2}}{4 \eta}\right)^{2}+H(r)
$$

For $L>L_{c}$, the descent is expected to be monotonous while it should be oscillatory for $L<L_{c}$. Equation 5 predicts $L_{c} \sim 10 \mathrm{~cm}$ for millimetric tubes, a distance that rapidly decreases with the viscosity. But $L_{c}$ is even more sensitive to the tube radius found to be the main parameter controlling the descent dynamics. While the critical length is several meters for $r=1.5 \mathrm{~mm}$, it is only a few centimetres for $r=0.5 \mathrm{~mm}$.

In order to establish a dynamical phase diagram of capillary descent, we perform experiments with a collection of fifteen vertical tubes with different lengths and radii $(5<L(\mathrm{~cm})<13,0.5<r(\mathrm{~mm})<1.5)$. We report in figure 4 whether the meniscus oscillates (green data) or not (red data), and compare our observations with equation 5. The agreement is satisfactory, even if the transition to the oscillatory regime is slightly overestimated, which might reflect the existence of other sources of damping such as the singular pressure loss at the tube exit (if the liquid descends) or entrance (if the liquid rises), which we discuss in the SI (figure SI-4).

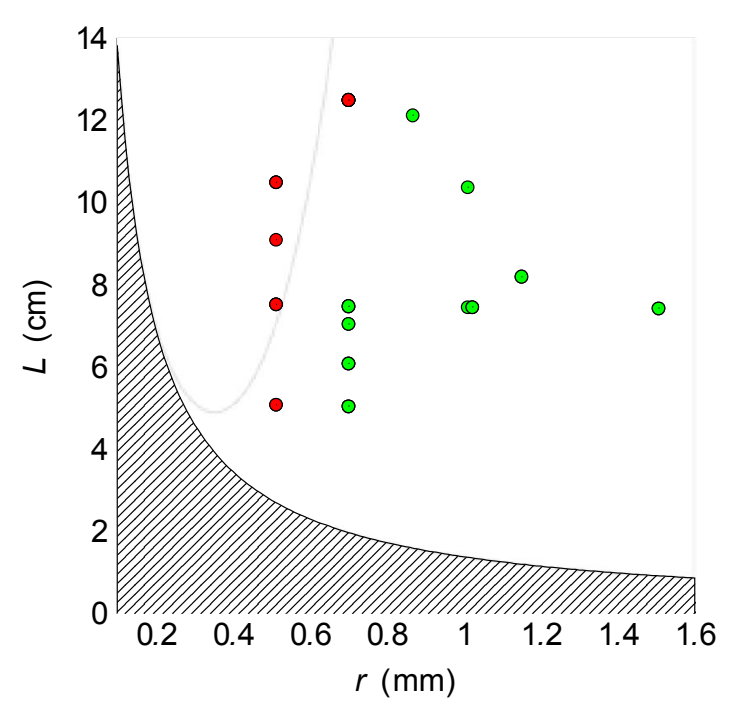

Figure 4. Dynamical phase diagram of capillary descent in the $(L, r)$ space. The green and red regions respectively indicate whether oscillations are expected or not from equation 5 . The hatched region stands for tubes smaller than the equilibrium depth, so that the meniscus pins at the tube end. Dots are the data (green dots: oscillating behaviour, red dots: no oscillation). 
The regime of monotonous descent is only observed for long and thin tubes, but the latter condition is not sufficient. As seen in figure 4, oscillations may also occur in thin tubes, provided that $L$ is smaller than typically $5 \mathrm{~cm}$, corresponding to the minimum of the function $L_{c}(r)$ in equation 5. Hence the minimal length required to observe monotonous regimes is $L_{\min } \approx \kappa^{-4 / 5} D^{1 / 5}$ where $D=\gamma^{2} / g \eta^{2}$ is a kilometric distance for water, making $L_{\min }$ a few centimetres, as reported in figure 4.

In summary, capillary descent provides a broad variety of dynamics, where the different liquid parameters (surface tension, viscosity, density) all play a role, owing to the combination of capillarity, viscous effects and inertia at stake in these experiments. It would be interesting to extend our results to the case of immersed hydrophobic porous media to see how our findings resist the reduction of scale and the complexity encountered in such materials. More generally, it is worth realizing that the inversion of the two fluids present in capillary rise generates similar equilibrium conditions, but quite different dynamics, due to the contrast in viscosity between water and air.

\section{References}

[1] P.S. de Laplace, Supplément à la théorie de l'action capillaire, Courcier, Paris, 1807.

[2] J.M. Bell and F.K. Cameron, "The flow of liquids through capillary spaces," J. Phys. Chem., 50, 658-674, 1906.

[3] R. Lucas, "Ueber das Zeitgesetz des kapillaren Aufstiegs von Flüssigkeiten," KolloidZeitschrift, 23, 15-22, 1918.

[4] E. W. Washburn, "The dynamics of capillary flow," Phys. Rev., 17, 273-283, 1921.

[5] E. Guyon, J.P. Hulin, L. Petit, and C.D. Mitescu, Physical Hydrodynamics, 2nd ed., Oxford University Press, 2015.

[6] D. Deng, Y. Tang, J. Zeng, S. Yang, and H. Shao, "Characterization of capillary rise dynamics in parallel micro V-grooves," Int. J. Heat Mass Transf., 77, 311-320, 2014.

[7] A. Shen, Y. Liu, X. Qiu, Y. Lu, and S. Liang, "A model for capillary rise in nano-channels with inherent surface roughness," Appl. Phys. Lett., 110, 121601, 2017.

[8] A. Perwuelz, P. Mondon, and C. Caze, "Experimental study of capillary flow in yarns," Text. Res. J., 70, 333-339, 2000.

[9] M. Lago and M. Araujo, "Capillary rise in porous media," J. Colloid Interface Sci., 234, 3543, 2001.

[10] A. Marmur, "Kinetics of penetration into uniform porous media: Testing the equivalentcapillary concept," Langmuir, 19, 5956-5959, 2003.

[11] S.F. Nia and K. Jessen, "Theoretical Analysis of Capillary Rise in Porous Media," Transp. Porous Media, 110, 141-155, 2015.

[12] A. Marmur and R.D. Cohen, "Characterization of porous media by the kinetics of liquid penetration: the vertical capillaries model," J. Colloid Interface Sci., 189, 299-304, 1997.

[13] J. Bachmann, S.K. Woche, M.O. Goebel, M.B. Kirkham, and R. Horton, "Extended methodology for determining wetting properties of porous media," Water Resour. Res., 39, 1-14, 2003.

[14] T.L. Staples and D.G. Shaffer, "Wicking flow in irregular capillaries," Colloids Surfaces A 
Physicochem. Eng. Asp., 204, 239-250, 2002.

[15] M. Reyssat, L. Courbin, E. Reyssat, and H. A. Stone, "Imbibition in geometries with axial variations," J. Fluid Mech., 615, 335-344, 2008.

[16] B. Figliuzzi and C.R. Buie, "Rise in optimized capillary channels," J. Fluid Mech., 731, 142161, 2013.

[17] L. Romero and F. Yost, "Flow in an open channel capillary," J. Fluid Mech, 322, 109-129, 1996.

[18] M. M. Weislogel, “Compound capillary rise,” J. Fluid Mech., 709, 622-647, 2012.

[19] F.J. Higuera, A. Medina, and A. Liñán, "Capillary rise of a liquid between two vertical plates making a small angle," Phys. Fluids, 20, 102102, 2008.

[20] A. Ponomarenko, D. Quéré, and C. Clanet, "A universal law for capillary rise in corners," J. Fluid Mech., 666, 146-154, 2011.

[21] J. Kim, J. Ha, and H.-Y. Kim, “Capillary rise of non-aqueous liquids in cellulose sponges," J. Fluid Mech., 818, R2, 2017.

[22] D. Quéré, "Inertial capillarity," Europhys. Lett., 39, 533-538, 1997.

[23] N. Fries and M. Dreyer, "The transition from inertial to viscous flow in capillary rise," J. Colloid Interface Sci., 327, 125-128, 2008.

[24] J. Jurin, "An account of some new experiments relating to the action of glass tubes upon water and quicksilver," Philos. Trans. R. Soc. London, 30, 1083-1096, 1717.

[25] H. de Maleprade, C. Clanet, and D. Quéré, "Spreading of bubbles after contacting the Lower side of an aerophilic slide immersed in water," Phys. Rev. Lett., 117, 094501, 2016.

[26] J. Wang, Y. Zheng, F. Q. Nie, J. Zhai, and L. Jiang, "Air bubble bursting effect of lotus leaf," Langmuir, 25, 14129-14134, 2009.

[27] B.V. Zhmud, F. Tiberg, and K. Hallstensson, "Dynamics of capillary rise," J. Colloid Interface Sci., 228, 263-269, 2000. 